\title{
Circulating Current Suppression Strategy of Modular Multilevel Converter
}

\author{
Xiu-Juan MA \\ Dept. Electrical Engineering \\ Harbin Institute of Technology at Weihai \\ Weihai, China \\ Hua-Qiang Zhang \\ Dept. Electrical Engineering \\ Harbin Institute of Technology at Weihai \\ Weihai, China
}

\author{
Jia-Yi Teng \\ Dept. Electrical Engineering \\ Harbin Institute of Technology at Weihai \\ Weihai, China \\ Tong Yao \\ Dept. Electrical Engineering \\ Harbin Institute of Technology at Weihai \\ Weihai, China
}

\begin{abstract}
Output voltage waveforms of modular multilevel converter (MMC) with less harmonics and high quality by switching the sub-modules, it becomes a hot research in the field of voltage source converter DC transmission. The basic topology structure and working principle of MMC are introduced, the mechanism of MMC circulating current is analyzed, and point out the necessity of controlling. To utilize the carrier phase-shift modulation (CPSM), circulating current suppression strategy based on quasi-proportional resonant (quasi-PR) control is proposed to suppress circulating current and keep voltage stability of sub-module(SM) capacitor. The effectiveness and feasibility of the proposed method is proved by Matlab/Simulink, the simulation results show that the circulating current can be confined in a small range.
\end{abstract}

Keywords-Modular Multilevel Converter; Circulating Current Suppression; Capacitor Voltage Balancing; Carrier Phase-Shift Modulation

\section{INTRODUCTION}

In 1990, the Canadian scholar Boon-Teck Ooi proposed high-voltage direct current transmission technology based on voltage source converter (VSC-HVDC) ${ }^{[1]}$. VSC-HVDC has no short circuit capacity requirements, it can supply power to passive network and dispense with filter, it can quickly and independently adjust active and reactive power. Therefore, VSC-HVDC has a wide range of application prospects in renewable energy grid generation, rural power grid transformation, urban network expansion and regional power grid interconnection ${ }^{[2-3]}$. The voltage source converter is the core component of the flexible direct current transmission system.

In 2001, the german scholar Rainer Marquardt proposed modular multilevel converter (MMC). MMC has a common DC bus, which will help to achieve back-to-back system. MMC has the same modular structure, it can generate multilevel waveforms, the output waveform has less harmonic content and high-quality waveforms. At the same time, MMC has good fault ride-through and protection capabilities ${ }^{[4-5]}$. So MMC become a potential converter technology for high voltage direct current (HVDC) transmission system.

At present, the researches on MMC mainly focus on topological structure, modulation strategy, capacitor-voltage balance control and circulating current suppression etc. The basic topology structure, working principle and circulating current in $\alpha \beta$ coordinate system are analyzed in this paper. In view of the problem of circulating current, circulating current suppression strategy of modular multilevel converter is proposed to suppress circulating current and keep voltage stability of sub-module(SM) capacitor. The effectiveness and feasibility of the proposed method is proved by Matlab/Simulink.

\section{MODULAR MULTILEVEL CONVERTER}

The modular multilevel converter (MMC) topology is shown in Fig. 1, which consists of three strict symmetrical phase units, each phase unit is divided into the upper and lower two arms, each arm is cascaded by $\mathrm{N}$ sub-module and an inductor. The function of the inductor is to inhibit the phase circulation caused by the fluctuation of sub-module capacitor voltage. Each sub-module consists of a half-bridge unit and a DC capacitor, the sub-module structure is shown in Fig. 2.

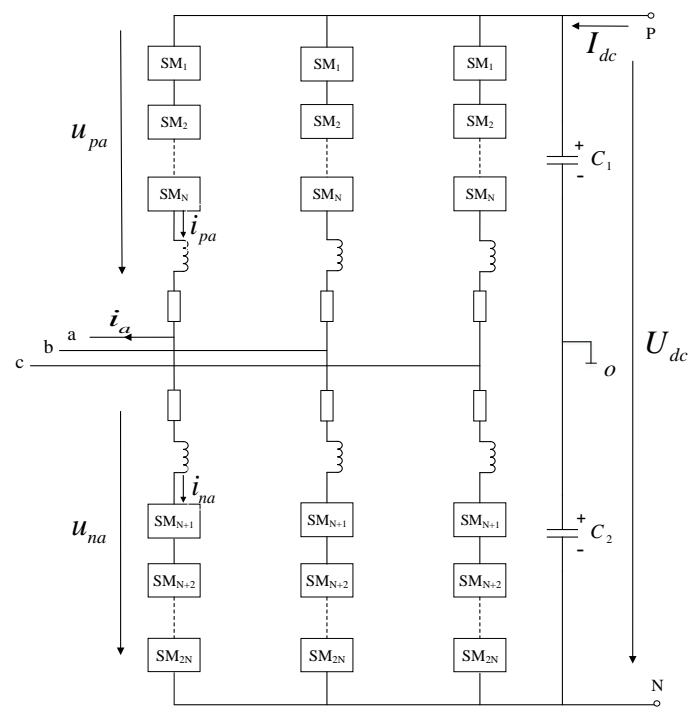

Fig. 1. Topology structure of MMC 


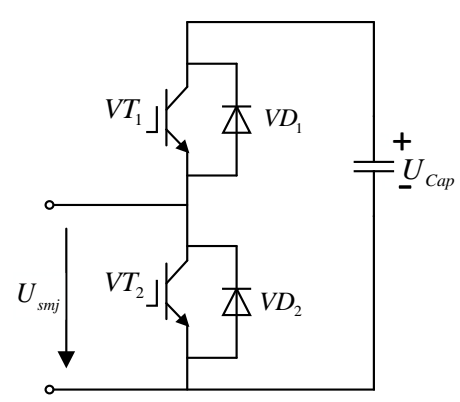

Fig. 2. Configuration of Sub-module

Taking a-phase as an example, o is the midpoint of the DC side potential of the inverter, assuming that the output voltage of the AC side is $u_{a o}$. It is the same potential between the o point and the equivalent midpoint of the three-phase voltage, then the upper and lower arm voltage respectively are:

$$
\left\{\begin{array}{l}
u_{p a}=\frac{1}{2} U_{d c}-u_{a o}-L \frac{d i_{p a}}{d t}-R i_{p a} \\
u_{n a}=\frac{1}{2} U_{d c}+u_{a o}-L \frac{d i_{p a}}{d t}-R i_{p a}
\end{array}\right.
$$

The upper and lower arm current respectively are:

$$
\left\{\begin{array}{l}
i_{p a}=\frac{i_{a}}{2}+i_{z a} \\
i_{n a}=-\frac{i_{a}}{2}+i_{z a}
\end{array}\right.
$$

Circulating current expressed with formula as follows:

$$
i_{z a}=\frac{1}{2}\left(i_{p a}+i_{n a}\right)
$$

The sub-modules input number of the upper and lower arm vary according to the sine law, resulting in the step sinusoidal $\mathrm{AC}$ voltage waveform. The more modules, the more the level, the output closer to sine wave.

\section{ANALYSIS OF CIRCUlating CURRENT}

In order to propose a more accurate method of circulating current suppression, the mechanism of MMC circulating current is analyzed. MMC is a symmetrical structure, take a phase as an example, AC output voltage and current respectively are

$$
\left\{\begin{array}{l}
u_{a}(\mathrm{t})=\frac{k}{2} U_{d c} \sin w_{0} t \\
i_{a}(\mathrm{t})=I_{a} \sin \left(w_{0} t+\varphi\right)
\end{array}\right.
$$

By the energy conservation of DC and AC side can get

$$
\begin{aligned}
i_{z a}= & \frac{u_{a} i_{a}}{U_{d c}} \\
= & \frac{\frac{k}{2} U_{d c} \sin w_{0} t \cdot I_{a} \sin \left(w_{0} t+\varphi\right)}{U_{d c}} \\
= & \frac{k}{4} I_{a} \cos \varphi-\frac{k}{4} I_{a} \cos \left(2 w_{0} t+\varphi\right)
\end{aligned}
$$

As can be seen from (5), the circulating current $i_{z a}$ mainly includes the DC component and the double fundamental frequency component.

By the active power balance of DC and AC side can get

$$
3 U I \cos \varphi=U_{d c} I_{d c}
$$

Where, $U$ and $I$ respectively are virtual value of AC output, Udc and Idc respectively are voltage and current of DC side.

Substituting (4) into (6) can get

$$
k I_{a} \cos \varphi=\frac{4}{3} I_{d c}
$$

The DC component of the circulating current is

$$
\frac{k}{4} I_{a} \cos \varphi=\frac{1}{3} I_{d c}
$$

As can be seen from (8), when the three-phase AC system is balanced, the DC component of the circulating current is one-third of the DC current, That is, at this time, the DC side current of MMC is evenly distributed among the three-phases.

So the upper and lower arm current respectively are:

$$
\left\{\begin{array}{l}
i_{p a}=\frac{i_{a}}{2}+i_{z a}=\frac{i_{a}}{2}+\frac{I_{d c}}{3}+i_{2 f} \\
i_{n a}=-\frac{i_{a}}{2}+i_{z a}=-\frac{i_{a}}{2}+\frac{I_{d c}}{3}+i_{2 f}
\end{array}\right.
$$

So circulating current can be expressed as

$$
i_{z a}=\frac{1}{2}\left(i_{p a}+i_{n a}\right)=\frac{I_{d c}}{3}+i_{2 f}
$$

The circulation path of the circulating current is shown in Fig. 3. 


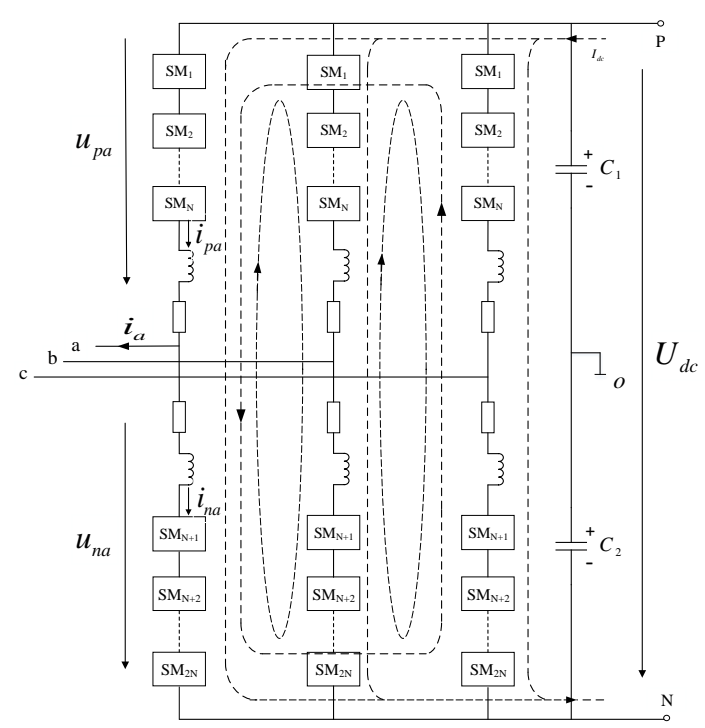

Fig. 3. Three-phase circulating current circulation path

The existence of MMC circulating current increases the unnecessary loss and distorts the arm current, and the fluctuation of sub-module capacitor voltage is too large, resulting in output current and voltage waveforms do not achieve the expected results ${ }^{[6-7]}$. Therefore, it is necessary to suppress the circulating current.

\section{CIRCULATING CURRENT SUPPRESSION STRATEGY}

The unbalanced voltage drop on the arm generated by aphase arm currents can be derived from Kirchhoff's law:

$$
u_{z a}=L \frac{d i_{z a}}{d t}+R i_{z a}
$$

Thus, the expression of three-phase unbalanced voltage drop generated by circulating current can be written:

$$
\left[\begin{array}{l}
u_{z a} \\
u_{z b} \\
u_{z c}
\end{array}\right]=L \frac{d}{d t}\left[\begin{array}{l}
i_{z a} \\
i_{z b} \\
i_{z c}
\end{array}\right]+R\left[\begin{array}{l}
i_{z a} \\
i_{z b} \\
i_{z c}
\end{array}\right]
$$

Equation (12) through Clark transformation can get:

$$
\left\{\begin{array}{l}
u_{z \alpha}=L \frac{d i_{z \alpha}}{d t}+R i_{z \alpha} \\
u_{z \beta}=L \frac{d i_{z \beta}}{d t}+R i_{z \beta}
\end{array}\right.
$$

Where, $i_{z \alpha}$ and $i_{z \beta}$ respectively are AC component of circulating current in $\alpha \beta$ axis, $u_{z \alpha}$ and $u_{z \beta}$ respectively are AC component of three-phase unbalanced voltage drop in $\alpha \beta$ axis. In the $\alpha \beta$ coordinate system, $i_{z \alpha}$ and $i_{z \beta}$ are independent of each other, the decoupling control is not needed, and the design of the circulating current suppressor is convenient.

According to above analysis, this paper designs a circulating current suppressor based on quasi-PR control, as shown in Fig. 4.

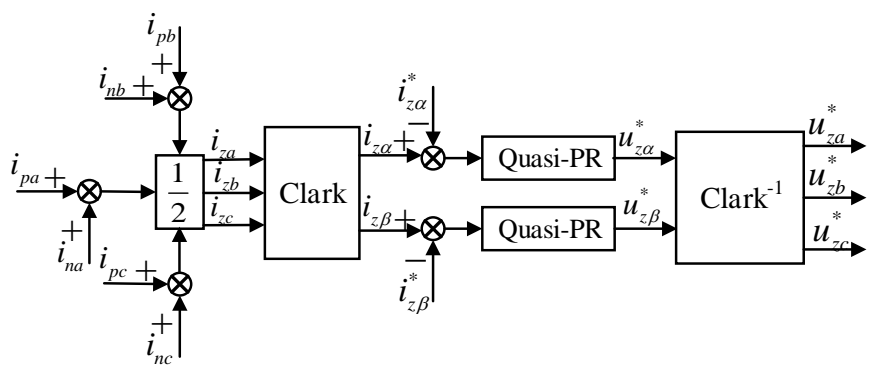

Fig. 4. Dynamic block diagram of circulating current suppression strategy

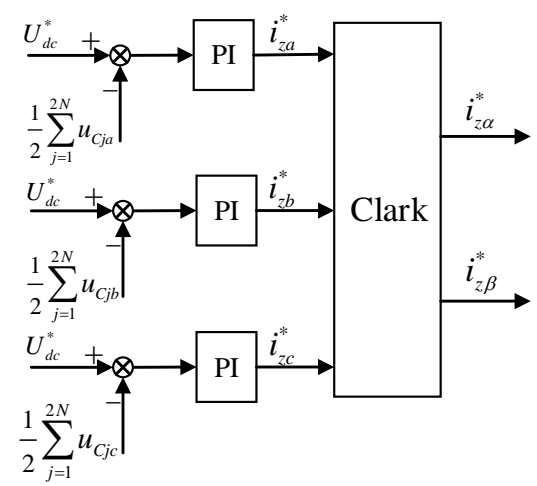

Fig. 5. Dynamic block diagram of circulating current reference value

The circulating current reference value of the controller is not 0 , but is obtained by PI control, as shown in Fig. 5. $U_{d c}$ track its reference value $U_{d c}^{*}, U_{d c}$ is obtained by adding submodules capacitor voltages, which not only suppress circulating current but also keep voltage stability of submodule(SM) capacitor. The actual value of the circulating current is obtained by the upper and lower arm currents. The reference value and the actual value of circulating current are subjected to Clark transformation to obtain the component on the $\alpha \beta$ axis, the actual circulating current values $i_{z \alpha}$ and $i_{z \beta}$ are compared with the reference values $i_{z \alpha}^{*}$ and $i_{z \beta}^{*}$, and then the circulating current is suppressed by quasi-PR controller. The output is the reference value of the unbalanced voltage drop on the $\alpha \beta$ axis, and then the reference value of the unbalanced voltage drop under the three-phase is obtained. And it is used as the regulating variable of the circulating current suppression.

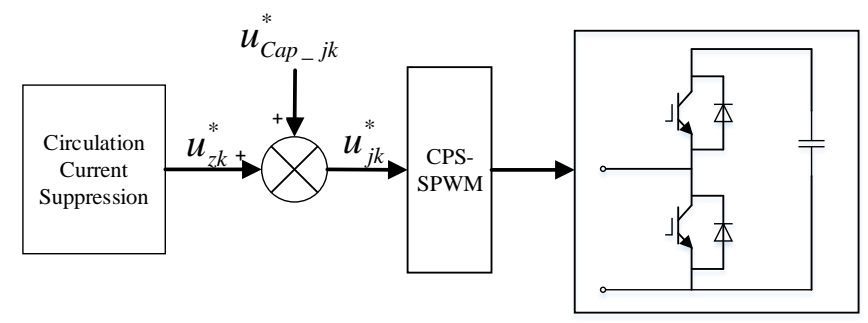

Fig. 6. Block diagram of MMC control

The MMC control structure is shown in Fig. $6, u_{j k}^{*}$ is the reference voltage corresponding to each sub-module, $u_{j k}^{*}$ is taken as the reference amplitude of the modulation wave, $N$ 
modulated waves with an amplitude of $u_{j k}^{*}$ are compared with $N$ triangular carriers with the same frequency and the same amplitude, but phase difference of $2 \pi / N$. Generating groups of $N$ PWM trigger signals, respectively controlling the put-into or cut-off of $N$ sub-modules.

\section{SIMULATION RESULT AND ANALYSIS}

In order to verify the effectiveness of the method, a 5-level MMC simulation model is built in MATLAB/Simulink, carrier frequency $f_{c}=2 \mathrm{kHz}$, sub-module capacitance $C=1.8 \mathrm{mF}$, arm inductance $L=6 \mathrm{mH}$, DC side voltage $U_{d c}=600 \mathrm{~V}$, sub-module capacitor voltage reference value $u_{C a p}=150 \mathrm{~V}$. The simulation results are shown in Fig. 7-14.

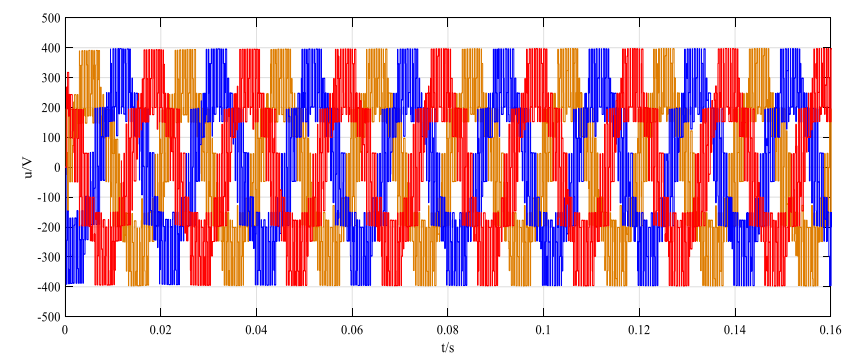

Fig. 7. Three-phase ac output voltage of MMC

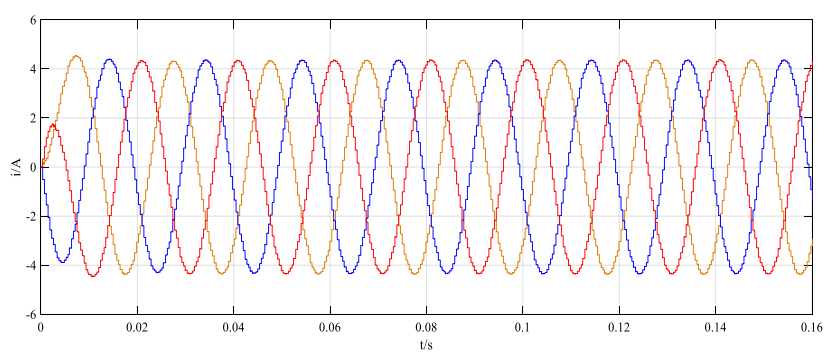

Fig. 8. Three-phase ac output current of MMC

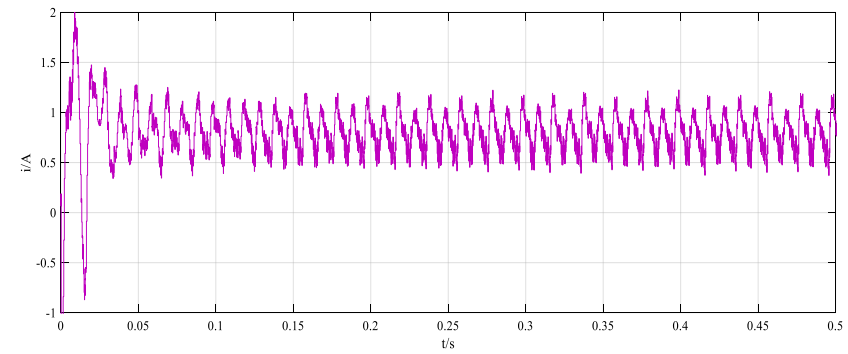

Fig. 9. a-phase circulating current

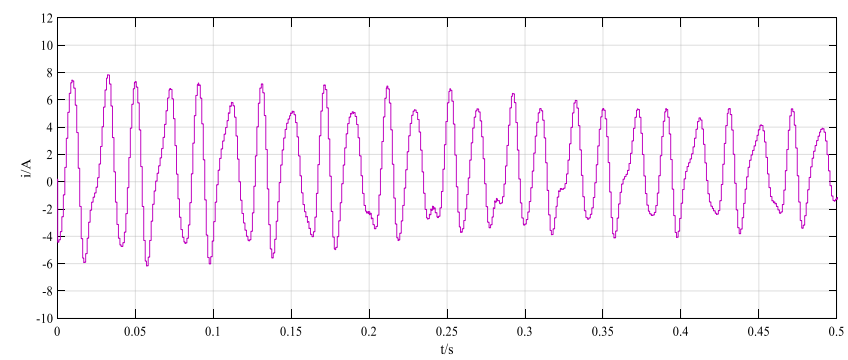

Fig. 10. a-phase circulating current(without circulating current suppression)

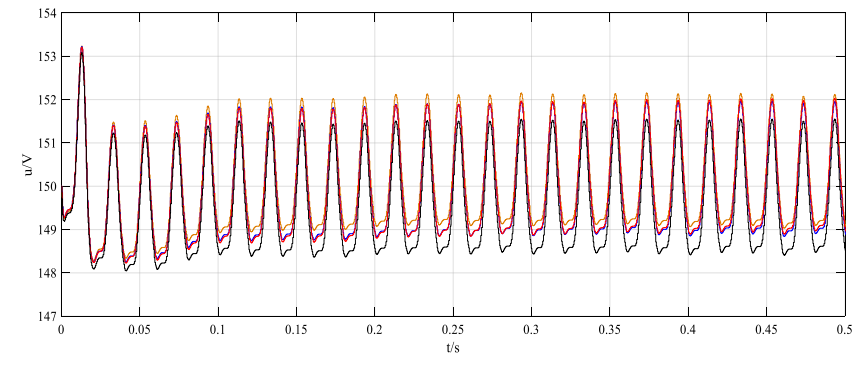

Fig. 11. Sub-module capacitor voltage in upper arm of a-phase

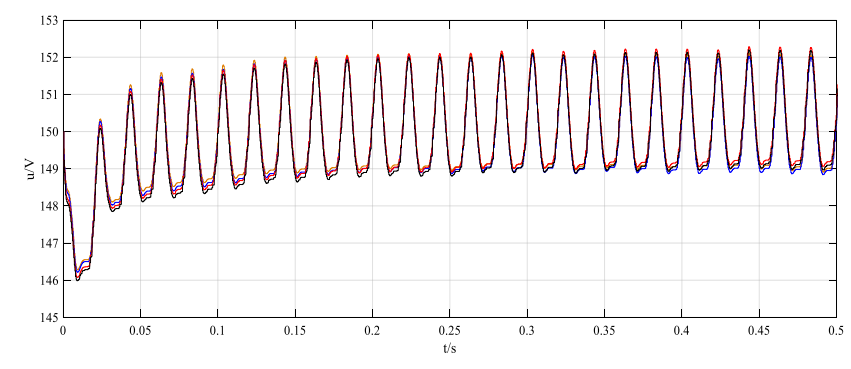

Fig. 12. Sub-module capacitor voltage in lower arm of a-phase

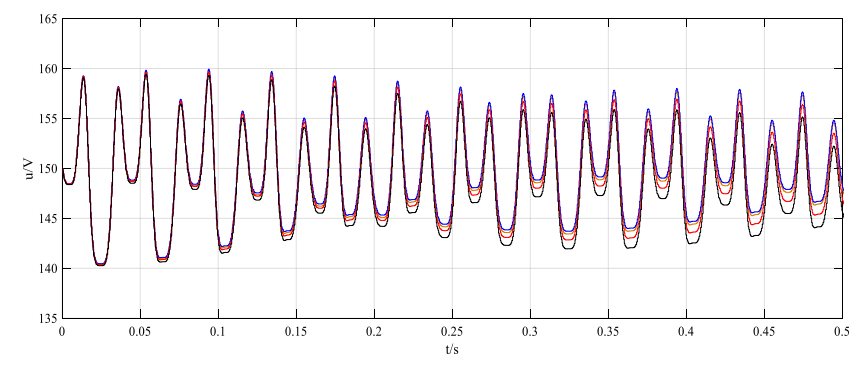

Fig. 13. Sub-module capacitor voltage in upper arm of a-phase(without circulating current suppression)

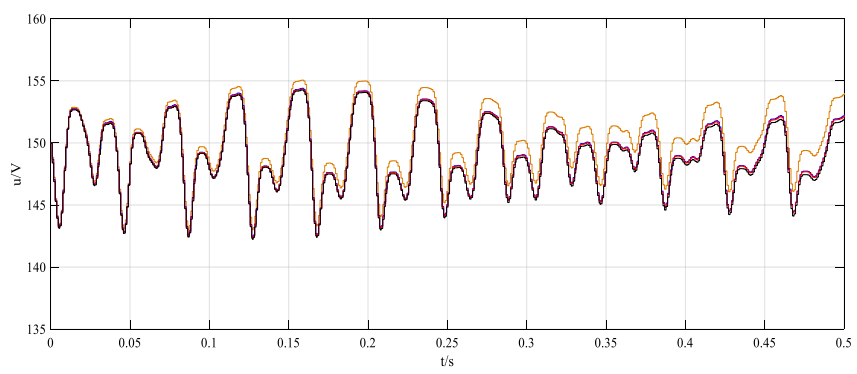

Fig. 14. Sub-module capacitor voltage in lower arm of a-phase(without circulating current suppression)

Fig. 7 is the phase voltage of the MMC three-phase AC output, and Fig. 8 is three-phase AC output current. From the simulation waveform, it can be seen that the three-phase AC output voltage and the output current are close to the sine wave. Fig. 10 is a-phase circulating current waveform without the circulating current suppression, the circulating current fluctuates between -4 6A, Fig. 9 is a-phase circulating current waveform after the circulating current suppression, the circulating current fluctuates between $0.5 \sim 1.3 \mathrm{~A}$, only about 8 $\%$ of the original. It shows that the circulating current is suppressed. Fig. 11 and Fig. 12 respectively are the capacitor voltage waveform of the four sub-module of a-phase the upper and lower arm after the circulating current suppression. Fig. 13 and Fig. 14 respectively are the capacitor voltage waveform of the four sub-module of a-phase the upper and lower arm 
without the circulating current suppression. It can be seen from the figure that when there is no suppression strategy, the capacitor voltage of each sub-module fluctuates greatly around the reference value of $150 \mathrm{~V}$, which is not conducive to the DC side voltage stability. The capacitor voltage conversion trend of the four sub-modules is consistent after circulating current suppression. The voltage fluctuates around the reference value $150 \mathrm{~V}$, and the positive and negative fluctuation range are controlled within $3 \%$, thus ensuring the DC bus voltage stability. The simulation results show that circulating current suppression strategy of modular multilevel converter is beneficial to suppress circulating current and keep voltage stability of sub-module(SM) capacitor. It verifies the correctness and effectiveness of the proposed method.

\section{CONCLUSION}

MMC is considered as the most potential topology in the field of high-voltage power conversion in the future. At present, it has been applied in several high-voltage high-power projects, especially in the field of flexible DC transmission. Since Siemens AG of Germany took the lead in building the Trans Bay Cable project in 2010, there are dozens of flexible DC transmission projects under construction or planned to be constructed adopting the structure of MMC in domestic and foreign. In China, the flexible DC transmission project of Nanhui, Nanao, Zhoushan, Xiamen and Luxi is VSC - HVDC project based on $\mathrm{MMC}^{[8]}$. MMC as a popular multilevel converter, stable operation is the key technology of its application. In this paper, circulating current suppression strategy of modular multilevel converter is proposed by utilizing the CPSM. A five-level MMC simulation model is built on the MATLAB/Simulink platform, the simulation results show that the control method can suppress the circulating current and stabilize the capacitor voltage.

\section{ACKNOWLEDGMENT}

The authors thank the support both the Foundation of National Natural Science (51377168) and the Foundation of Shandong Province Natural Science (ZR2017MEE053), which enabled the achievement of the mentioned research results.

\section{REFERENCES}

[1] Chengyong Li, Jianzhong $\mathrm{Xu}$, and Tan $\mathrm{Li}$, "DC transmission modeling technology of Modular Multilever Converter," Beijing: China Electric Power Press, 2017, pp. 1-4.

[2] Dandan Xu, Suppression of Circulating Current Harmonics and Capacitor Voltage Fluctuations in Modular Multilevel Converters, Harbin: Harbin Institute of Technology, 2015, pp.13.

[3] Xinhang Xie, Research on control strategy of MMC-HVDC, Beijing: Beijing Jiaotong University, 2017, pp. 1-8.

[4] Kaijing Liu, Suppression Strategy on Bridge Arm Circulation of MMC-STATCOM in Three-Phase Four Wire System, Beijing: North China Electric Power University, 2017, pp. 4-5.

[5] Ao Li, Research on voltage control and circulation suppression strategy of Modular Multilevel Converter, Wuhan: Huazhong University of Science \& Technology, 2015, pp. 4-7.

[6] Decun Niu, Zhongwei Sun, Nan Li, Yanli Li, Wei Tang, and Lei Zhao, "Study on MMC-HVDC New Type Loop Current Control Strategy Based on Quasi-PR Controller," Power Capacitor \& Reactive Power Compensation, vol. 37, pp. 87-94, August 2016.

[7] Jinyu Wang, Jun Liang, Chengfu Wang, and Xiaoming Dong, "Circulating current suppression for MMC-HVDC under unbalanced grid conditions," IEEE Transactions on Industry Applications, vol. 53, pp. 3250-3259, August 2017.

[8] Qiang Song and Hong Rao, "Analysis and design of the converters in VSC-HVDC system," Beijing: Tsinghua University Press, 2014, pp. 4-8. 